\title{
Vision-based Landing of Fixed-wing Miniature Air Vehicles
}

Blake Barber

Timothy McLain

Brigham Young University - Provo, mclain@byu.edu

Barrett Edwards

Follow this and additional works at: https://scholarsarchive.byu.edu/facpub

Part of the Mechanical Engineering Commons

\section{Original Publication Citation}

Duncan Blake Barber, Tim McLain, and Barrett Edwards. "Vision-Based Landing of Fixed-Wing Miniature Air Vehicles". Journal of Aerospace Computing Information and Communication 03/ 2009; 6(3):1-20. DOI: 10.2514/1.36201

\section{BYU ScholarsArchive Citation}

Barber, Blake; McLain, Timothy; and Edwards, Barrett, "Vision-based Landing of Fixed-wing Miniature Air Vehicles" (2007). Faculty Publications. 1501.

https://scholarsarchive.byu.edu/facpub/1501

This Peer-Reviewed Article is brought to you for free and open access by BYU ScholarsArchive. It has been accepted for inclusion in Faculty Publications by an authorized administrator of BYU ScholarsArchive. For more information, please contact ellen_amatangelo@byu.edu. 


\title{
Vision-Based Landing of Fixed-Wing Miniature Air Vehicles
}

\author{
Blake Barber Timothy McLain* Barrett Edwards \\ Brigham Young University Provo, UT 84602
}

\begin{abstract}
This paper outlines a method for using vision-based feedback to accurately land a MAV on a visually identifiable target of approximately known location. The method presented is robust to wind, capable of handling both stationary and moving targets, and capable of correcting for camera misalignment, state estimation biases, and parameter estimation biases. Landing results from actual flight tests are presented which demonstrate the effectiveness of the proposed method.
\end{abstract}

\section{Introduction}

Fixed-wing miniature air vehicles (MAVs) have garnered much attention recently for their usefulness in both civil and military applications. MAVs have primarily been deployed as short range aerial reconnaissance vehicles. In this role MAVs are used to collect actionable information which is then passed on to other agents that must spend time navigating to the target site. In many such instances it would be desirable to have the MAV take action immediately rather than waiting for other agents.

An example of such a scenario is a search and rescue mission. Typically MAVs would be used to quickly comb a search area, locating the lost individual and directing search teams into the target region. However, in many instances it may be beneficial to take immediate action rather than waiting for ground-based agents. For instance, some of the searching MAVs may carry food, medicine, or a communication device such as a two-way radio. It would be desirable to deliver this payload accurately to a target region in plain sight of the lost individual.

Another such mission is convoy protection, in which a team of MAVs is used to identify and monitor threats to a convoy moving through hostile terrain. Once a threat is identified it is desirable to neutralize the threat as quickly as possible. The MAV which has been used to identify the threat is uniquely positioned to take action. Once again it is desirable to have the MAV deliver a payload as accurately as possible into a specified target area.

For the purposes of this paper we will refer to missions in which the MAV is used to search for and deliver a payload to a target site as search and engage missions. This paper will focus on the engagement part of this mission. In previous work we have demonstrated the ability to use hand-launched MAVs to geolocate objects of interest to within 2 to 5 meters in high wind conditions. ${ }^{1}$ In more recent testing the same methods have been used to estimate both target position and velocity with high accuracy. In this work it will be assumed that the engaging MAV has access to measurements of target position and velocity from a searching MAV. These measurements will be used to assist the engaging MAV to accurately deliver an attached payload to the target site. Accurate landing at the target site makes use of the noisy position and velocity estimates initially, but then uses vision-based control once the target has been acquired in the engaging MAV's field of view.

The problem of accurately landing a UAV using vision-based control has been well studied for autonomous rotorcraft. ${ }^{2-5}$ Most of these approaches utilize structure from motion algorithms and require a structured landing environment. A method that does not require a structured landing environment has been developed by researchers at the Army/NASA Rotorcraft Division. ${ }^{6,7}$

Techniques for the landing of rotorcraft, however, rely on the ability to hover and thus do not transition well to fixed-wing platforms. Significantly less work exists for using visual sensors to accurately land fixedwing aircraft. Those techniques which have been developed generally require a set of known landmarks

${ }^{*}$ Corresponding author, email: mclain@byu.edu 
or some visually identifiable pattern. ${ }^{8,9}$ The vision-based landing method developed in this work does not require the presence of any such landmarks.

An alternative vision-based approach for accurately landing fixed-wing aircraft without the need for landmarks involves the use of a dedicated visual sensor for measuring optic flow. $\mathrm{In}^{10}$ Chahl et al. demonstrated that by maintaining constant optic flow during a landing maneuver, a MAV's descent could be successfully controlled. Development of lightweight sensors for measurement of optic flow ${ }^{11-13}$ has further bolstered this approach. Barrows et al. have demonstrated that these sensors can be successfully used to follow terrain in low flying MAVs. ${ }^{14}$

This work builds on prior work done at BYU in which vector-field based control is combined with optic flow measurements to accurately land a MAV. ${ }^{15}$ This work will use the landing algorithm outlined in ${ }^{15}$ to guide the approach of the MAV until the target is visually acquired. At this point the control is turned over to the vision-based controller outlined in the remainder of this paper.

The vision-based control approach is preferred to the use of dedicated optic flow sensors because it transitions better to search and engage missions: It allows for moving targets, and it is not affected by errors in the initial estimate of target location. The approach to vision-based control taken in this paper combines a feedback loop based on the observed pixel coordinates of the target with a bias estimator and application of counterbiases. This control scheme is shown schematically in Figure 1

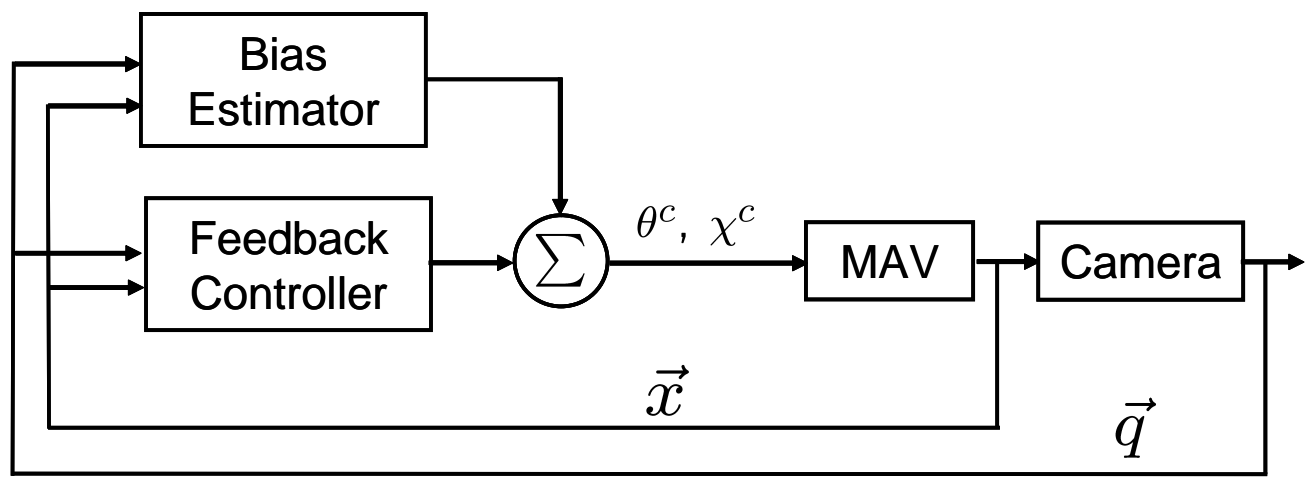

Figure 1. Diagram of the control scheme outlined in this paper.

The remainder of this paper is organized as follows: Section II contains a description of the longitudinal control, focusing on the effects of bias errors on the performance of the control law. Section III discusses the effect of wind and introduces a method for accounting for wind in the longitudinal control. Section IV explains how to account for moving targets using a "pseudo-wind" and the methods outlined in Section III. Section V extends the ideas developed for longitudinal control to lateral control. Section VI combines the lateral and longitudinal control laws. Section VII outlines the addition of adaptive counter-bias terms to the feedback control law developed in Section VI. Section VIII gives hardware results demonstrating successful landing on both stationary and moving targets. Finally, the paper ends with conclusions in Section IX.

\section{Two-Dimensional Model for Longitudinal Control}

For the purposes of this paper the MAV is assumed to be either durable or expendable and the survivability of the MAV is not considered. Any of a range of glideslopes that intersect with the target are considered acceptable regardless of the resulting impact velocity. This allows us to choose the nominal desired glideslope to be linear rather than the asymptotic glideslope that is typical for the landing of fixed-wing aircraft. In addition, deviations from the nominal linear glideslope which may increase impact velocity will be allowed and considered acceptable provided the deviant glideslope intersects the target. In practice, the impact velocity of the aircraft can still be controlled by appropriately selecting the nominal linear glideslope. However, selection of the nominal glideslope to enhance MAV survivability is airframe dependent and is not addressed.

Accurately tracking a linear glideslope requires aligning the motion vector of the MAV with the position vector $\mathbf{p}$ extending from the MAV to the target. A unit vector in the direction of $\mathbf{p}$, denoted by $\mathbf{u}_{\mathbf{p}}$, can be found using the pixel coordinates of the target in the image and the coordinate transformations outlined 
in. ${ }^{1}$ Once $\mathbf{u}_{\mathbf{p}}$ has been determined, all that remains is to align the MAV's motion vector. However, both the process of calculating $\mathbf{u}_{\mathbf{p}}$ and aligning the MAVs motion vector with $\mathbf{u}_{\mathbf{p}}$ are subject to bias errors due to camera misalignment as well as parameter and state estimation errors. It is important to understand the effects of these biases on landing accuracy.

To examine these effects, consider the following simplified two-dimensional model for the longitudinal dynamics of the MAV in the absence of wind:

$$
\begin{aligned}
& \dot{x}=V_{a} \cos (\theta-\alpha) \\
& \dot{h}=V_{a} \sin (\theta-\alpha) .
\end{aligned}
$$

In (1) and (2) $h$ and $x$ are defined by the earth-fixed coordinate frame shown in Figure 2, $V_{a}$ is the airspeed of the MAV, $\theta$ is the pitch of the MAV, and $\alpha$ is the angle of attack of the MAV. $V_{a}$ is treated as an input to the system and is maintained constant. $\theta$ is also treated as an input to the system and is set according to the control law

$$
\theta^{c}=\hat{\gamma}_{t g t}+\hat{\alpha},
$$

where $\hat{\gamma}_{t g t}$ represents the estimate of the angle between the ray from the MAV to the target, and the ground as shown in Figure 2, and $\hat{\alpha}$ represents the estimate of the MAV's angle of attack.

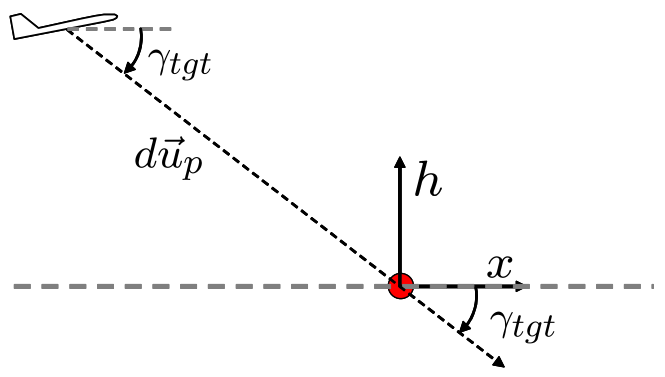

Figure 2. Two-dimensional longitudinal coordinate system.

The value of $\hat{\gamma}_{t g t}$ is given by the four quadrant inverse tangent of the components of $\mathbf{u}_{\mathbf{p}}$ plus the relative orientation estimation error $\tilde{\gamma}_{t g t}$. That is

$$
\hat{\gamma}_{t g t}=\operatorname{atan} 2\left(u_{p h}, u_{p x}\right)+\tilde{\gamma}_{t g t} .
$$

Similarly, the value of $\hat{\alpha}$ is given by

$$
\hat{\alpha}=\alpha+\tilde{\alpha}
$$

where $\tilde{\alpha}$ represents the parameter estimation error.

Substituting Equations (4) and (5) into Equation (3) gives

$$
\theta^{c}=\operatorname{atan} 2\left(u_{p h}, u_{p x}\right)+\alpha+\tilde{\gamma}_{t g t}+\tilde{\alpha} .
$$

Combining the error terms and replacing them with the new variable $\tilde{\theta}$ gives

$$
\theta^{c}=\operatorname{atan} 2\left(u_{p h}, u_{p x}\right)+\alpha+\tilde{\theta} .
$$

Replacing $\theta$ in the dynamic model given by Equations (1) and (2) with this expression for $\theta^{c}$ gives

$$
\begin{aligned}
& \dot{x}=V_{a} \cos \left(\operatorname{atan} 2\left(u_{p h}, u_{p x}\right)+\tilde{\theta}\right) \\
& \dot{h}=V_{a} \sin \left(\operatorname{atan} 2\left(u_{p h}, u_{p x}\right)+\tilde{\theta}\right),
\end{aligned}
$$

The combined effect of parameter estimation error, $\tilde{\alpha}$, and relative orientation estimation error, $\tilde{\gamma}_{t g t}$, can be seen by integrating the equations of motion given in (8) and (9) for different values of $\tilde{\theta}$. Trajectories corresponding to different values of $\tilde{\theta}$ are shown in Figure 3 for two different nominal glideslopes. 


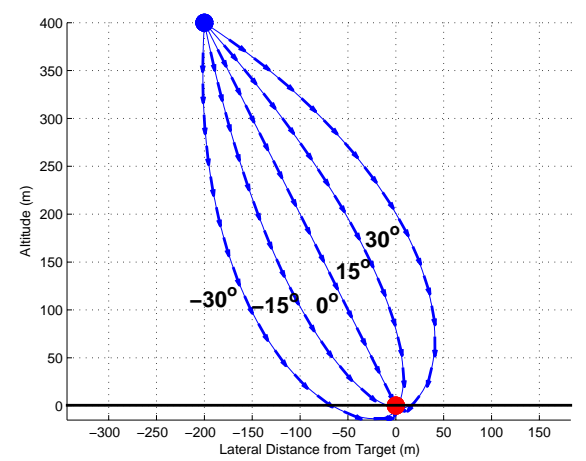

(a) Steep Nominal Glideslope

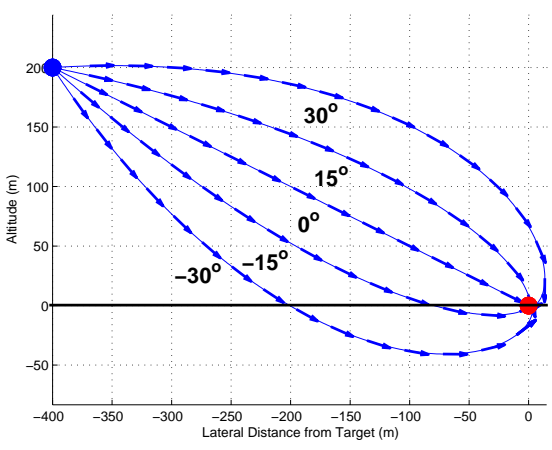

(b) Shallow Nominal Glideslope

Figure 3. Effects of nominal glideslope on landing error. In each case the shallowest trajectory corresponds to $\tilde{\theta}=-30^{\circ}$. The steepest trajectory corresponds to $\tilde{\theta}=30^{\circ}$. The intermediate trajectories represent fifteen degree increments in $\tilde{\theta}$.

It can be seen in Figure 3 that errors in correctly aligning the motion vector of the MAV with the unit vector $\mathbf{u}_{\mathbf{p}}$ can cause overshoot or undershoot in the landing location. It is interesting to note, however, that relatively large amounts of error can be tolerated without significant effect on landing position. The sign and magnitude of the error which can be tolerated is a function of the nominal glideslope.

For the case of the steeper nominal glideslope shown in Figure 3(a), the landing trajectory associated with $\tilde{\theta}=-30^{\circ}$ results in a landing error ${ }^{\mathrm{a}}$ of approximately $65 \mathrm{~m}$. For the case of the more shallow nominal glideslope shown in Figure 3(b), the landing trajectory associated with the same value of $\tilde{\theta}$ results in a landing error of approximately $200 \mathrm{~m}$. However, for $\tilde{\theta}=30^{\circ}$ the shallow glideslope gives better results with an overshoot error of approximately $10 \mathrm{~m}$ compared to approximately $45 \mathrm{~m}$ for the steep glideslope.

The preceding analysis indicates that it may be advantageous to bias the control either positive or negative depending on the magnitude of the nominal glideslope (shallow glideslopes benefit from positive bias whereas steep glideslopes benefit from negative bias). The magnitude of the desired bias depends on the error satistics of the bias term $\tilde{\theta}$. The total misalignment due to the unknown value of $\tilde{\theta}$ can be biased either positive or negative through the addition of the bias term, $\bar{\theta}$. Making this change to the longitudinal control law in Equation (3) gives

$$
\theta^{c}=\hat{\gamma}_{t g t}+\hat{\alpha}+\bar{\theta}
$$

The value of $\bar{\theta}$ is chosen based on the desired shift in the distribution from which the value of $\tilde{\theta}$ is realized.

\section{Effects of Wind}

The analysis in Section II assumes that the wind speed is negligible. In this case, the glideslope angle relative to a coordinate frame fixed to the mass of moving air, $\gamma_{a}$, is the same as the glideslope angle relative to a coordinate frame fixed to the ground, $\gamma_{g}$. Although this is not generally the case, this simplifying assumption can be made when the wind speed represents a small percentage of the vehicle's airspeed. However, for MAVs this is rarely the case. MAVs typically operate in conditions where the wind speed is $10 \%$ to $60 \%$ or more of the commanded airspeed. For this reason, the effects of wind must be accounted for by the control law.

To see the effects of wind, we examine again the simplified two-dimensional longitudinal control law presented in Section II. For this analysis we assume a constant wind with a component only in the $x$ direction with magnitude $V_{w}$. We can then rewrite the dynamic model given by Equations (1) and (2) as

$$
\begin{aligned}
\dot{x} & =V_{a} \cos (\theta-\alpha)+V_{w} \\
\dot{h} & =V_{a} \sin (\theta-\alpha) .
\end{aligned}
$$

${ }^{a}$ For undershoot landing error is defined as the distance between the point where the landing trajectory intersects with the ground plane and the desired landing point. For overshoot the landing error is defined as the distance between the maximum $x$ value of the landing trajectory and the desired landing point. 
The goal of the controller is to align the motion vector of the MAV with the estimate of the unit vector in the direction from the MAV to the target, $\hat{\mathbf{u}}_{\mathbf{p}}$. In other words it is desired to set $\gamma_{g}$ equal to $\hat{\gamma}_{\text {tgt }}$. This can be done by appropriately choosing the value of $\gamma_{a}$ and setting the input $\theta^{c}$ to achieve this value. Towards this end, the relationships shown in Equations (11) and (12) can be rewritten as the vector equation

$$
\mathbf{V}_{\mathrm{g}}=\mathbf{V}_{\mathbf{a}}+\mathbf{V}_{\mathbf{w}}
$$

where

$$
\begin{aligned}
& \mathbf{V}_{\mathbf{g}}=\left[\begin{array}{ll}
\dot{x} & \dot{h}
\end{array}\right]^{\top}, \\
& \mathbf{V}_{\mathbf{a}}=\left[\begin{array}{ll}
V_{a} \cos (\theta-\alpha) & V_{a} \sin (\theta-\alpha)
\end{array}\right]^{\top}, \\
& \mathbf{V}_{\mathbf{w}}=\left[\begin{array}{ll}
V_{w} & 0
\end{array}\right]^{\top} .
\end{aligned}
$$

This vector equation is represented graphically in Figure 4.

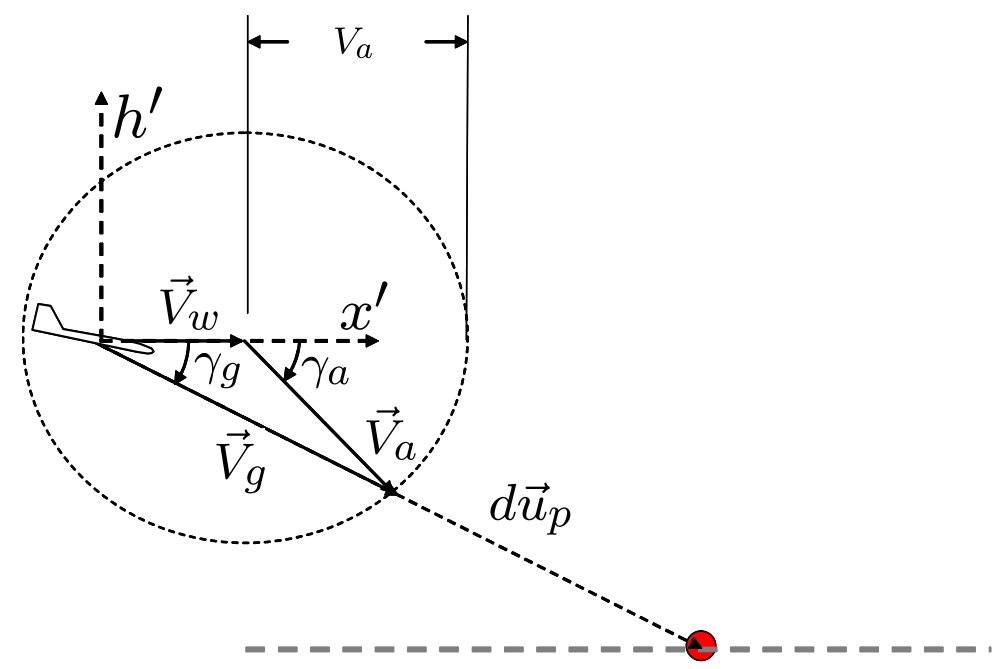

Figure 4. Graphic representation of the vector relationship described in Equation (13). The dotted circle has its center at the tip of the wind vector and radius equal to $V_{a}$.

The goal is to align the desired groundspeed vector $\mathbf{V}_{\mathbf{g}}^{\mathbf{d}}$ with $\hat{\mathbf{u}}_{\mathbf{p}}$. The estimate of the wind vector is given by $\hat{\mathbf{V}}_{\mathbf{w}}$ and the magnitude of the airspeed vector $V_{a}$ is an input to the system that is held constant at a known value. The desired groundspeed vector is related to the known quantities by the relationship given in Equation (13).

Aligning the desired groundspeed vector $\mathbf{V}_{\mathbf{g}}^{\mathbf{d}}$ with $\hat{\mathbf{u}}_{\mathbf{p}}$ requires finding the value of $\gamma_{a}^{d}$ which causes Equation (13) to be satisfied subject to the constraint

$$
\mathbf{V}_{\mathbf{g}}^{\mathbf{d}}=V_{g}^{d} \hat{\mathbf{u}}_{\mathbf{p}}
$$

The value of $V_{g}^{d}$ can be determined by solving for the point of intersection of a line that is collinear with $\hat{\mathbf{u}}_{\mathbf{p}}$, and a circle of radius $V_{a}$ centered at the tip of the wind vector. This is represented graphically in Figure 4.

For convenience, we solve for this point of intersection in terms of a coordinate frame attached to the center of mass of the MAV. In this coordinate frame the equation for any vector coincident with $\hat{\mathbf{u}}_{\mathbf{p}}$ is given by the vector equation

$$
\left[\begin{array}{c}
x^{\prime} \\
h^{\prime}
\end{array}\right]=d_{1} \hat{\mathbf{u}}_{\mathbf{p}}=\left[\begin{array}{c}
d_{1} \cdot \hat{u}_{p x} \\
d_{1} \cdot \hat{u}_{p h}
\end{array}\right],
$$

where $\hat{u}_{p x}$ and $\hat{u}_{p h}$ represent the $x$ and $h$ components of $\hat{\mathbf{u}}_{\mathbf{p}}$, and $d_{1}$ is arbitrary. The equation for the circle is given by

$$
\left(x^{\prime}-\hat{V}_{w}\right)^{2}+h^{\prime 2}=V_{a}^{2} .
$$


The point of intersection can be found by replacing $x^{\prime}$ and $h^{\prime}$ in Equation (19) with the expressions shown in Equation (18). This gives

$$
\left(d_{1} \hat{u}_{p x}-\hat{V}_{w}\right)^{2}+d_{1}^{2} \hat{u}_{p h}^{2}=V_{a}^{2} .
$$

Equation (20) can be solved by finding the positive root of this quadratic equation in the variable $d_{1}$. This is given by

$$
d_{1}=\hat{u}_{p x} \hat{V}_{w}+\sqrt{V_{a}^{2}\left(\hat{u}_{p x}^{2}+\hat{u}_{p h}^{2}\right)-\hat{u}_{p h}^{2} \hat{V}_{w}^{2}} .
$$

The value of $d_{1}$ given by (21) represents the magnitude of the desired groundspeed vector, $V_{g}^{d}$. Substituting $d_{1}$ into the constraint equation given in (17) we get

$$
\mathbf{V}_{\mathbf{g}}^{\mathbf{d}}=d_{1} \hat{\mathbf{u}}_{\mathbf{p}} .
$$

Once $\mathbf{V}_{\mathbf{g}}^{\mathbf{d}}$ has been determined, the desired airspeed vector $\mathbf{V}_{\mathbf{a}}^{\mathbf{d}}$ can be found by simple vector arithmetic

$$
\mathbf{V}_{\mathbf{a}}^{\mathbf{d}}=\mathbf{V}_{\mathrm{g}}^{\mathbf{d}}-\hat{\mathbf{V}}_{\mathbf{w}} .
$$

$\gamma_{a}^{d}$ can then be determined from the components of $\mathbf{V}_{\mathbf{a}}^{\mathbf{d}}, V_{a x}^{d}$ and $V_{a h}^{d}$, using the relationship

$$
\gamma_{a}^{d}=\operatorname{atan} 2\left(V_{a h}^{d}, V_{a x}^{d}\right)
$$

Finally, the control law given in Equation (10) can be rewritten in terms of $\gamma_{a}^{d}$ instead of $\hat{\gamma}_{t g t}$

$$
\theta^{c}=\gamma_{a}^{d}+\hat{\alpha}+\bar{\theta} .
$$

It is important to note that in addition to the relative orientation errors and parameter estimation errors that affected $\hat{\gamma}_{t g t}, \gamma_{a}^{d}$ is also affected by wind estimation errors. This becomes a consideration when selecting the magnitude of the bias term, $\bar{\theta}$.

\section{Effect of Moving Targets}

If the reference frame in which $x$ and $h$ are defined is taken to be that of the reference frame attached to the moving target, the effect of target motion is exactly the same as the effect of wind. As such, target motion can be modeled as an additional "pseudo-wind". In this case, target motion in the positive $x$ direction (away from the MAV) can be modeled as a pseudo-wind in the negative $x$ direction (headwind). Similarly, target motion in the negative $x$ direction (toward the MAV) can be modeled as a pseudo-wind in the positive $x$ direction (tailwind). This leads to the following expression for pseudo-wind

$$
\overline{\mathbf{V}}_{\mathbf{w}}=\left[\begin{array}{ll}
\left(\hat{V}_{w}-\hat{\dot{x}}_{t g t}\right) & 0
\end{array}\right]^{\top} .
$$

The pseudo-wind, $\overline{\mathbf{V}}_{\mathbf{w}}$, can be used in place of $\hat{\mathbf{V}}_{\mathbf{w}}$ in the calculations outlined in Section III. This results in a controller that is able to track and land on constant-velocity targets as well as stationary targets. It is important to note that in this case the term $\gamma_{a}^{d}$ will be affected by target velocity estimation errors as well as the other error sources outlined above. Once again, this becomes a consideration when selecting the magnitude of $\bar{\theta}$.

\section{Two-Dimensional Model for Lateral Control}

Up to this point the analysis has dealt strictly with the two-dimensional model for longitudinal control. The extension of these same ideas to two-dimensional lateral control is straightforward. To see this, consider the following model for the lateral dynamics of the MAV in wind

$$
\begin{aligned}
& \dot{x}=V_{g} \cos \chi \\
& \dot{y}=V_{g} \sin \chi .
\end{aligned}
$$

These equations are relative to the coordinate frame shown in Figure 5. $\chi$ is the course of the MAV measured clockwise from the positive $x$ axis and is treated as an input to the system. $V_{g}$ is not a direct input to the system, but is determined by our choice of $V_{a}$ and the wind vector $\mathbf{V}_{\mathbf{w}}$. 


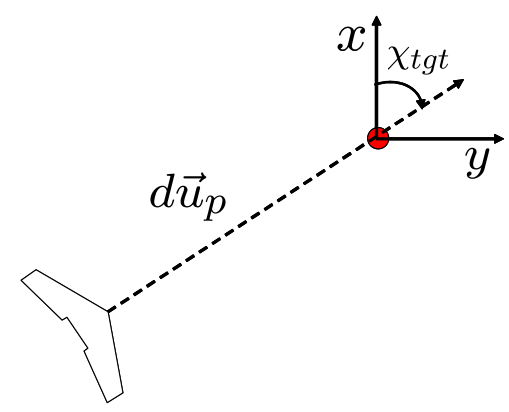

Figure 5. Two-dimensional lateral coordinate system.

As with the longitudinal control, the goal is to align the motion vector of the MAV with the estimate of the unit vector in the direction from the MAV to the target, $\hat{\mathbf{u}}_{\mathbf{p}}$. Because the orientation of the motion vector $(\chi)$ is an input to the system, this is trivial. The control law is simply

$$
\chi^{c}=\hat{\chi}_{t g t},
$$

where $\hat{\chi}_{t g t}$ is the estimate of the compass heading from the MAV to the target. The value of $\hat{\chi}_{t g t}$ is given by

$$
\hat{\chi}_{t g t}=\operatorname{atan} 2\left(u_{p y}, u_{p x}\right)+\tilde{\chi}_{t g t},
$$

where $\tilde{\chi}_{t g t}$ represents the relative orientation estimation error in the lateral plane.

An important distinction between the lateral and longitudinal controllers is the effect of parameter estimation errors. Rather than being subject to both relative orientation estimation error and parameter estimation error, the lateral control is only subject to relative orientation estimation error. This error will cause the same type of spiral deviations from the nominal linear trajectory as those shown in Figure 3 . For the longitudinal control we introduced the bias term $\bar{\theta}$ because there was an advantage to having either an upward or downward spiral depending on the nominal desired glideslope. However, no such advantages or disadvantages exist for having a right-hand versus a left-hand spiral. As such, the lateral control does not benefit from the addition of a similar bias term.

Another important distinction between the lateral control and the longitudinal control is the effect of wind. Unlike the longitudinal control, wind has no effect on the lateral control. This stems from the fact that the lateral equations of motion are written in terms of the system input $\chi$. Because $\chi$ is measured relative to a ground-based frame of reference it is unaffected by wind. However, this is not true for the pseudo-wind introduced in Section III to account for moving targets. The pseudo-wind needs to be accounted for in both the lateral and longitudinal control laws. The method for taking the pseudo-wind into account in the lateral control law will be covered in Section VI.

\section{Feedback Control Law}

The analysis thus far has dealt strictly with the two-dimensional cases of longitudinal and lateral control. This section extends these same ideas to three dimensions to form the feeback control law represented in Figure 1. The extension of these ideas into three dimensions with simultaneous longitudinal and lateral control is straightforward.

First, we rewrite the equations of motion in three dimensions using the target-centered coordinate frame shown in Figure 6. For convenience we will continue to write the equations in terms of MAV altitude $h$ using the change of variables $h=-z$. The equations of motion then become

$$
\begin{aligned}
\dot{x} & =V_{a} \cos (\theta-\alpha) \cos (\psi)+V_{w x} \\
\dot{y} & =V_{a} \cos (\theta-\alpha) \sin (\psi)+V_{w y} \\
\dot{h} & =V_{a} \sin (\theta-\alpha) .
\end{aligned}
$$

These equations of motion, however, are written in terms of MAV heading, $\psi$, rather than course, $\chi$, which is the input to the system. It is desired to rewrite these equations of motion in terms of the inputs, $V_{a}, \chi$, 


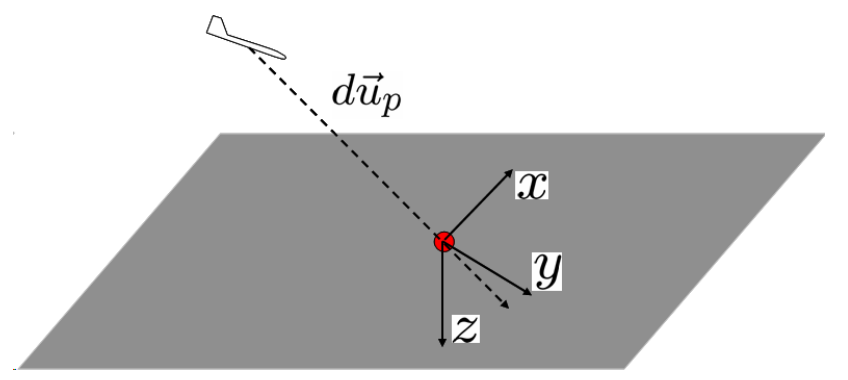

Figure 6. Three-dimensional coordinate system ( $x$ is north, $y$ is east, $z$ is down).

and $\theta$. Since the equations of motion are already in terms of $\theta$ and $V_{a}$ we focus on the lateral (xy) plane. The wind triangle that relates $\psi$ to $\chi$ is defined in the lateral plane as shown in Figure 7 .

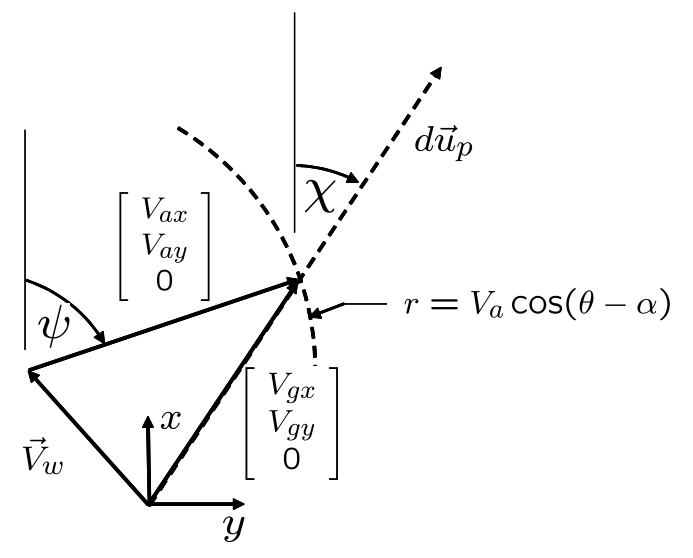

Figure 7. Wind triangle in the lateral $(x y)$ coordinate plane. Two sides and one angle of the triangle are known.

It is desired to solve the wind triangle represented in Figure 7 for the orientation of the airspeed vector, $\psi$, in terms of the system inputs: $V_{a}, \chi$, and $\theta$. The known quantities are the wind vector, $\mathbf{V}_{\mathbf{w}}$, the magnitude of the airspeed vector in the $x y$ plane, $V_{a} \cos (\theta-\alpha)$, and the direction of the groundspeed vector, $\chi$. The wind triangle can be solved by ray-circle intersection as outlined in Section III. In this case the unit vector is given by

$$
\left[\begin{array}{lll}
u_{p x} & u_{p y} & 0
\end{array}\right]^{\top}=\left[\begin{array}{lll}
\cos (\chi) & \sin (\chi) & 0
\end{array}\right]^{\top} .
$$

The point of intersection is found by projecting along the vector $\left[\begin{array}{lll}u_{p x} & u_{p y} & 0\end{array}\right]^{\top}$ by $d_{2}$, where $d_{2}$ is given by

$$
d_{2}=V_{w x} c_{\chi}+V_{w y} s_{\chi}+\sqrt{V_{w x}^{2} c_{\chi}^{2}+V_{w x} V_{w y} c_{\chi} s_{\chi}+V_{w y}^{2} s_{\chi}^{2}+\left(V_{a} c_{(\theta-\alpha)}\right)^{2}-V_{w x}^{2}-V_{w y}^{2}},
$$

and $c_{x}$ and $s_{x}$ refer to $\cos (x)$ and $\sin (x)$ respectively. In this case the value of $d_{2}$ represents the magnitude of the projection of the groundspeed vector onto the lateral plane,

$$
d_{2}=V_{g} \cos \left(\gamma_{g}\right)
$$

The value of $\psi$ can be determined from the point of intersection as

$$
\psi=\operatorname{atan} 2\left[d_{2} \sin (\chi)-V_{w y}, d_{2} \cos (\chi)-V_{w x}\right] .
$$

Substituting this expression into the equations of motion given in Equations (31) through (33) gives the equations of motion in terms of the control inputs 


$$
\begin{aligned}
& \dot{x}=V_{a} \cos (\theta-\alpha) \cos \left\{\operatorname{atan} 2\left[d_{2} \sin (\chi)-V_{w y}, d \cos (\chi)-V_{w x}\right]\right\}+V_{w x} \\
& \dot{y}=V_{a} \cos (\theta-\alpha) \sin \left\{\operatorname{atan} 2\left[d_{2} \sin (\chi)-V_{w y}, d \cos (\chi)-V_{w x}\right]\right\}+V_{w y} \\
& \dot{h}=V_{a} \sin (\theta-\alpha) . .
\end{aligned}
$$

In three dimensions the values for $\hat{\chi}_{t g t}$ and $\hat{\gamma}_{t g t}$ are given by:

$$
\begin{gathered}
\hat{\chi}_{t g t}=\operatorname{atan} 2\left(\hat{u}_{p y}, \hat{u}_{p x}\right) . \\
\hat{\gamma}_{t g t}=\sin ^{-1}\left(\hat{u}_{p h}\right) .
\end{gathered}
$$

Using Equations (41) and (42) to compute $\hat{\chi}_{t g t}$ and $\hat{\gamma}_{t g t}$, the control law given by Equations (10) and (29) can be used together to control the MAV for the case where wind speed is negligible (a rare case for MAVs).

The modification of the control law to account for the case where the wind speed is significant follows closely the analysis in Section III. As in Section III it is assumed that there is no wind component in the $z$ direction. This leads to a problem similar to that presented in Section III. The wind vector and the magnitude of the airspeed vector are known, and it is desired to find the airspeed vector that aligns the groundspeed vector with $\hat{\mathbf{u}}_{\mathbf{p}}$. In two dimensions this problem was solved by ray-circle intersection. In three dimensions this problem is solved by ray-sphere intersection. As with the $2 \mathrm{D}$ case, the ray is defined as a projection along the unit vector $\hat{\mathbf{u}}_{\mathbf{p}}$ of magnitude $d_{3}$. The sphere is centered at the tip of the wind speed vector and has a radius equal to $V_{a}$. The solution of the ray-sphere intersection problem proceeds similarly to that of the ray-circle intersection. The solution is given by

$$
\mathbf{V}_{\mathbf{g}}^{\mathbf{d}}=d_{3} \hat{\mathbf{u}}_{\mathbf{p}}
$$

where

$$
d_{3}=\hat{V}_{w x} \hat{u}_{p x}+\hat{V}_{w y} \hat{u}_{p y}+\sqrt{V_{a}^{2}-\hat{V}_{w x}^{2}\left(\hat{u}_{p y}^{2}+\hat{u}_{p h}^{2}\right)-\hat{V}_{w y}^{2}\left(\hat{u}_{p x}^{2}+\hat{u}_{p h}^{2}\right)-\hat{V}_{w x} \hat{V}_{w y} \hat{u}_{p x} \hat{u}_{p y}}
$$

Once $\mathbf{V}_{\mathbf{g}}^{\mathbf{d}}$ has been determined the desired airspeed vector can be calculated according to Equation (23). $\gamma_{a}^{d}$ can then be calculated from $\mathbf{V}_{\mathbf{a}}^{\mathbf{d}}$ as

$$
\gamma_{a}^{d}=\sin ^{-1}\left(\frac{V_{a h}^{d}}{\left\|\mathbf{V}_{\mathbf{a}}^{\mathbf{d}}\right\|}\right)
$$

Using this value for $\gamma_{a}^{d}$, Equations (29) and (25) can be used as the lateral and longitudinal control laws respectively. These control laws result in accurate landing at the target point so long as the errors, $\tilde{\theta}$ and $\tilde{\chi}$, are acceptably small and the target is stationary.

Moving targets can be accounted for using the pseudo-wind introduced in Section IV. In three dimensions pseudo-wind is defined as

$$
\overline{\mathbf{V}}_{\mathbf{w}}=\left[\begin{array}{lll}
\left(\hat{V}_{w x}-\hat{\dot{x}}_{t g t}\right) & \left(\hat{V}_{w y}-\hat{\dot{y}}_{t g t}\right) & 0
\end{array}\right]^{\top} .
$$

Introducing the new variable $\bar{d}$, and rewriting Equation (44) in terms of the components of the pseudo-wind, $\bar{V}_{w x}$ and $\bar{V}_{w y}$, gives

$$
\bar{d}=\bar{V}_{w x} \hat{u}_{p x}+\bar{V}_{w y} \hat{u}_{p y}+\sqrt{V_{a}^{2}-\bar{V}_{w x}^{2}\left(\hat{u}_{p y}^{2}+\hat{u}_{p h}^{2}\right)-\bar{V}_{w y}^{2}\left(\hat{u}_{p x}^{2}+\hat{u}_{p h}^{2}\right)-\bar{V}_{w x} \bar{V}_{w y} \hat{u}_{p x} \hat{u}_{p y}}
$$

Once $\bar{d}$ has been determined, it can be used to compute the pseudo-wind adjusted desired groundspeed vector, $\overline{\mathbf{V}}_{\mathbf{g}}^{\mathbf{d}}$, as

$$
\overline{\mathbf{V}}_{\mathbf{g}}^{\mathbf{d}}=\bar{d} \hat{\mathbf{u}}_{\mathbf{p}}
$$

The pseudo-wind adjusted desired airspeed vector $\overline{\mathbf{V}}_{\mathbf{a}}^{\mathbf{d}}$ can then be computed as

$$
\overline{\mathbf{V}}_{\mathbf{a}}^{\mathrm{d}}=\overline{\mathbf{V}}_{\mathrm{g}}^{\mathrm{d}}-\overline{\mathbf{V}}_{\mathbf{w}} \text {. }
$$


This value can then be used to compute the pseudo-wind corrected value of $\gamma_{a}^{d}$ as

$$
\bar{\gamma}_{a}^{d}=\sin ^{-1}\left(\frac{\bar{V}_{a h}^{d}}{\| \overline{\mathbf{V}}_{\mathbf{a}}^{\mathbf{d} \|}}\right) .
$$

Finally, the longitudinal control law given by (25) can be rewritten in terms of $\bar{\gamma}_{a}^{d}$ as

$$
\theta^{c}=\bar{\gamma}_{a}^{d}+\hat{\alpha}+\bar{\theta}
$$

Because the lateral control given by Equation (29) is insensitive to wind, it must be modified to take into account the moving target. The correct value of $\chi^{c}$ is given by the orientation of the groundspeed vector which results from tracking $\overline{\mathbf{V}}_{\mathbf{a}}^{\mathbf{d}}$ in the presence of the the estimated wind, $\hat{\mathbf{V}}_{\mathbf{w}}$ (not the pseudo-wind $\overline{\mathbf{V}}_{\mathbf{w}}$ ). This leads to the expression for the desired groundspeed vector

$$
\mathbf{V}_{\mathrm{g}}^{\mathrm{d}}=\overline{\mathbf{V}}_{\mathrm{a}}^{\mathrm{d}}+\hat{\mathbf{V}}_{\mathbf{w}} .
$$

The orientation of this vector in the lateral plane is given by

$$
\chi^{c}=\operatorname{atan} 2\left(\bar{V}_{a y}^{d}+\hat{V}_{w y}, \bar{V}_{a x}^{d}+\hat{V}_{w x}\right) .
$$

This represents the lateral control law that results in accurate landing on a moving target in the presence of wind. It is important to note that for the case where the pseudo-wind is equal to zero - meaning the target is stationary - this control law gives the same result as (29).

The trajectories resulting from integrating the equations of motion given in (38) through (40) subject to the longitudinal and lateral control laws given in Equations (51) and (53) are shown in Figure 8. Glideslopes are shown for values of $\tilde{\theta}$ and $\tilde{\chi}$ ranging from $-15^{\circ}$ each to $15^{\circ}$ each. It can be seen from Figure 8 that the same effects that were observed in the two-dimensional case are observed in the three-dimensional case. Errors cause the glideslope to deviate from the nominal linear glideslope, but relatively large errors can be tolerated without significantly affecting the landing point.

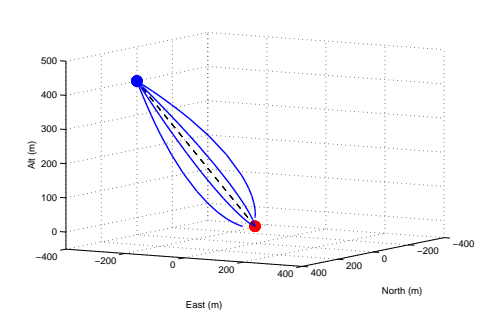

(a) Perspective View

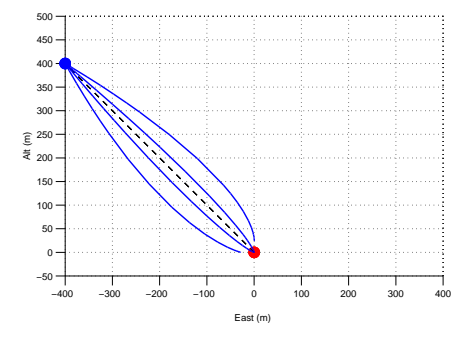

(b) Side View

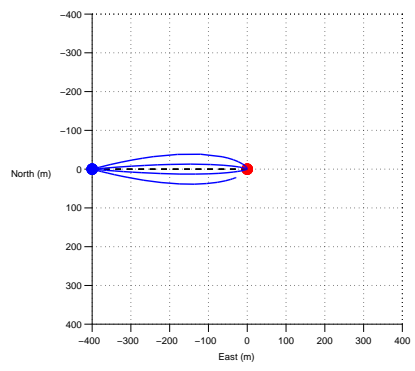

(c) Top View

Figure 8. The effect of errors, $\tilde{\theta}$ and $\tilde{\chi}$ on landing trajectory. The nominal linear glideslope is represented by the dashed line. The most shallow glideslope corresponds to $\tilde{\theta}=-15^{\circ}, \tilde{\chi}=-15^{\circ}$. The steepest glideslope corresponds to $\tilde{\theta}=15^{\circ}, \tilde{\chi}=15^{\circ}$. The intermediate glideslopes represent $10^{\circ}$ increments in each error term.

\section{Bias Correction}

To this point the analysis has focused on feedback control that does not correct for the effects of bias errors. However, significant bias error can result from a number of sources including relative orienation estimation error, parameter estimation error, target velocity estimation error, and wind estimation error. In the presence of these errors the feedback control law may still result in accurate landing at the target location. However, the trajectory followed to the target will deviate from the nominal linear trajectory. The amount of deviation is a function of the magnitude of the bias errors and can result in significant undershoot or overshoot. For this reason, it is desired to design a bias estimator that is able to estimate these biases. This bias estimator is represented schematically in Figure 1

Examination of each of the possible error sources leads to the definition of three different error terms. The first term, $\mu$, represents the error in tracking the desired linear trajectory. The second and third terms, 
$\nu_{\text {lat }}$ and $\nu_{\text {lon }}$, represent the error in correctly generating the desired trajectory. Each of these error terms is assumed to consist of a bias component as well as a Gaussian component. The goal of the bias estimator is to remove the bias component of the error while filtering the high-frequency noise content.

The first bias term, $\mu$, is caused by both parameter estimation errors and wind estimation errors. This tracking error affects only the longitudinal control, because the orientation of the MAV's lateral velocity vector $\chi$ is an input to the system. For the longitudinal control, however, the parameter $\alpha$ must be estimated in order to correctly choose $\theta^{c}$ given $\bar{\gamma}_{a}^{d}$ (see Equation 51). In addition, if the wind has not been estimated correctly then the lateral component of the actual groundspeed vector will be different than the anticipated lateral component of the groundspeed vector. At the same time the longitudinal component of the groundspeed vector remains $V_{a} \sin (\theta-\alpha)$. This results in an actual glideslope angle that deviates from the desired glideslope angle. The value of $\mu$ is defined as the difference between these glideslope angles. This value can be estimated using the desired groundspeed vector $\mathbf{V}_{\mathbf{g}}^{\mathbf{d}}$ and estimated actual groundspeed vector $\hat{\mathbf{V}}_{\mathbf{g}}^{\mathbf{a}}$ as

$$
\hat{\mu}=\sin ^{-1}\left(\frac{V_{g h}^{d}}{\left\|\mathbf{V}_{\mathbf{g}}^{\mathbf{d}}\right\|}\right)-\sin ^{-1}\left(\frac{\hat{V}_{g h}^{a}}{\left\|\hat{\mathbf{V}}_{\mathbf{g}}^{\mathbf{a}}\right\|}\right) .
$$

The second and third error terms, $\nu_{l a t}$ and $\nu_{l o n}$, represent the errors in correctly selecting the desired trajectory, $\mathbf{V}_{\mathbf{g}}^{\mathbf{d}}$. If the target is known to be stationary, these error terms are affected only by relative orientation estimation error. If the target is moving, these error terms are also affected by wind estimation errors and target velocity estimation errors. To see how this error term can be calculated, consider the longitudinal case shown in Figure 9. Given measurements at two different times, $t_{1}$ and $t_{2}$, we can develop
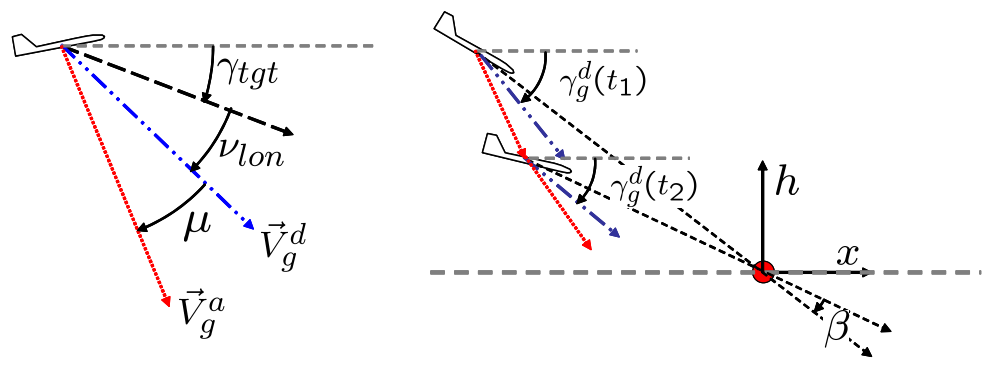

Figure 9. Two dimensional longitudinal tracking given the error terms $\mu$ and $\nu_{l o n}$

an approximate expression for $\nu_{l o n}$. First, we define $\nu_{l o n}$ in terms of $\gamma_{g}^{d}$ as

$$
\nu_{l o n}=\gamma_{g}^{d}-\gamma_{t g t}
$$

where $\gamma_{t g t}$ represents the orientation of the motion vector that, if tracked, would result in a linear landing trajectory. Next, we define the angle $\beta$ as shown in Figure 9. Given the assumption that the bias component of $\nu_{l o n}$ varies slowly in time, we can write an expression for $\beta$ as

$$
\beta \approx \gamma_{g}^{d}\left(t_{2}\right)-\gamma_{g}^{d}\left(t_{1}\right)
$$

where $\gamma_{g}^{d}$ is given by

$$
\gamma_{g}^{d}=\sin ^{-1}\left(\frac{V_{g h}^{d}}{\left\|\mathbf{V}_{\mathbf{g}}^{\mathbf{d}}\right\|}\right)
$$

and $\gamma_{g}^{d}(t)$ refers to the value of $\gamma_{g}^{d}$ at time $t$.

Introducing the term $\lambda$ to represent the distance to the target and $\kappa$ to represent the linear distance travelled by the MAV between times $t_{1}$ and $t_{2}$, we can write another approximation involving $\beta$

$$
\lambda \beta \approx \kappa \sin \left(\mu+\nu_{l o n}\right) .
$$

Substituting the value for $\beta$ given in Equation (56) into this expression and solving for $\nu_{l o n}$ gives

$$
\nu_{l o n} \approx \sin ^{-1}\left(\frac{\lambda\left(\gamma_{g}^{d}\left(t_{2}\right)-\gamma_{g}^{d}\left(t_{1}\right)\right)}{\kappa}\right)-\mu .
$$


In this expression the value of $\mu$ can be estimated using (54), and the values of $\lambda$ and $\kappa$ can be estimated from GPS. Thus Equation (59) can be rewritten in terms of known or measurable quantities as

$$
\hat{\nu}_{l o n}=\sin ^{-1}\left(\frac{\hat{\lambda}\left(\gamma_{g}^{d}\left(t_{2}\right)-\gamma_{g}^{d}\left(t_{1}\right)\right)}{\hat{\kappa}}\right)-\hat{\mu} .
$$

Similar arguments can be used to derive an approximation for $\nu_{\text {lat }}$. The main difference being that there is no $\mu$ component in the lateral direction for the reasons outlined above. In the lateral case the estimate of the error term is given by

$$
\hat{\nu}_{l a t}=\sin ^{-1}\left(\frac{\hat{\lambda}\left(\chi^{d}\left(t_{2}\right)-\chi^{d}\left(t_{1}\right)\right)}{\hat{\kappa}}\right) .
$$

Having developed expressions for estimating the error terms $\mu, \nu_{l a t}$, and $\nu_{l o n}$ from known or measurable quantities, we now use these values to define the bias correction terms $M, N_{\text {lat }}$, and $N_{\text {lon }}$. These bias correction terms represent filtered estimates of the error terms $\mu, \nu_{l a t}$, and $\nu_{l o n}$. These values are updated according to the differential equations

$$
\begin{aligned}
\dot{M} & =k_{1} \hat{\mu} \\
\dot{N}_{\text {lat }} & =k_{2} \hat{\nu}_{\text {lat }} \\
\dot{N}_{\text {lon }} & =k_{3} \hat{\nu}_{\text {lon }} .
\end{aligned}
$$

Correct selection of the gains $k_{1}, k_{2}$, and $k_{3}$ result in bias correction terms that adapt to the bias components of the error terms while attenuating the high frequency Gaussian component. These adaptive terms are then included in the control laws outlined in Section VI giving

$$
\begin{gathered}
\theta^{c}=\bar{\gamma}_{a}^{d}+\hat{\alpha}+\bar{\theta}+M+N_{l o n} \\
\chi^{c}=\operatorname{atan} 2\left(\bar{V}_{a y}^{d}+\hat{V}_{w y}, \bar{V}_{a x}^{d}+\hat{V}_{w x}\right)+N_{l a t} .
\end{gathered}
$$

This control functions such that so long as $\theta^{c}$ or $\chi^{c}$ result in trajectories that are nonlinear, the error terms are non-zero. As long as the error terms are non-zero, the values of $M, N_{l a t}$, and $N_{l o n}$ will adapt, thus forcing the commands closer to the commands that result in a linear trajectory.

The effect of adding these adaptive terms to the control law can be seen by integrating the equations of motion given by Equations (38) through (40) subject to the control laws given in (65) and (66). This can be seen in Figure 10 which compares the performance of the feedback control law with bias correction to that of the feedback control law without bias correction. The calculation of the control inputs was subject to the following bias errors:

- Relative Orienatation Estimation Error - Azimuth, $15^{\circ}$; Elevation, $15^{\circ}$

- Wind Estimation Error - Wind Speed, $3 \mathrm{~m} / \mathrm{s}$; Wind Direction, $30^{\circ}$

- Target Velocity Estimation Error — Target Speed, $3 \mathrm{~m} / \mathrm{s}$; Target Direction, $30^{\circ}$

- Parameter Estimation Error - $\alpha, 5^{\circ}$

The nominal conditions for this test were a wind speed of $4 \mathrm{~m} / \mathrm{s}$ from the northeast, a target speed of $4 \mathrm{~m} / \mathrm{s}$ to the southeast, and a commanded airspeed of $13 \mathrm{~m} / \mathrm{s}$.

The trajectories shown in Figure 10 demonstrate the ability of the bias correction to effectively reduce deviation from the nominal linear trajectory caused by bias errors. This allows larger magnitude bias errors to be tolerated by the system without significantly affecting landing accuracy.

\section{Hardware Results}

\section{A. Hardware Testbed}

BYU has developed a reliable and robust platform for testing unmanned air vehicles. ${ }^{16}$ Figure 11 shows the key elements of the testbed. The first frame shows the Procerus ${ }^{\mathrm{b}}$ Kestrel autopilot (originally developed at

\footnotetext{
${ }^{\mathrm{b}} \mathrm{http}: / /$ procerusuav.com/
} 


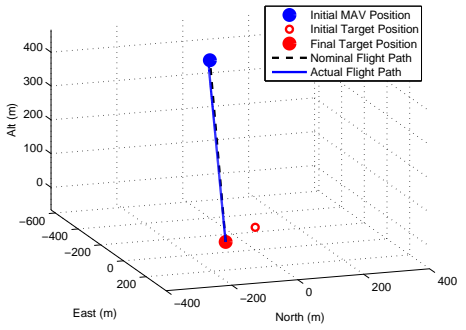

(a) 3D View - High Gain Bias Correction

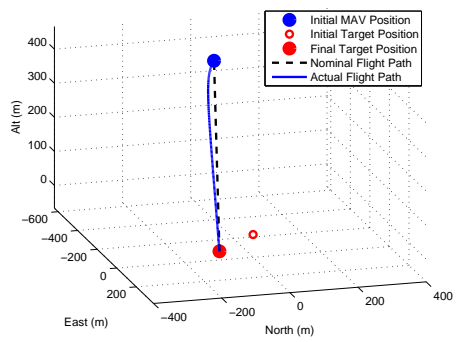

(d) 3D View - Low Gain Bias Correction

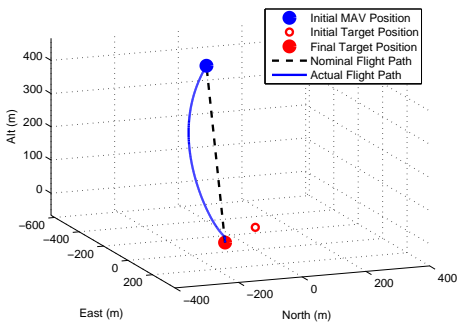

(g) 3D View - No Bias Correction

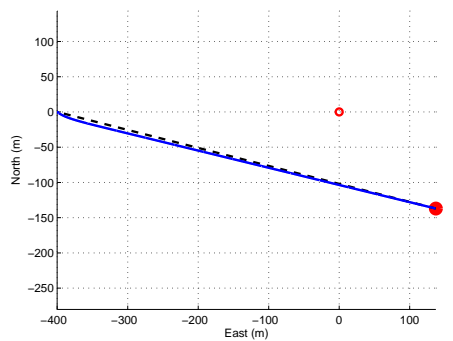

(b) Top View - High Gain Bias Correction

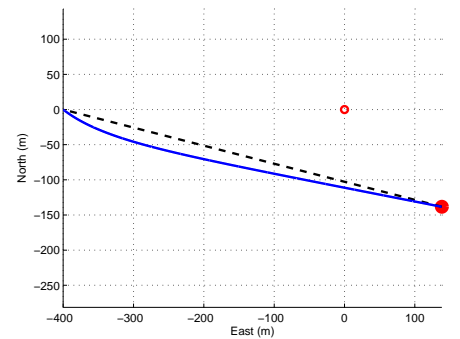

(e) Top View - Low Gain Bias Correction

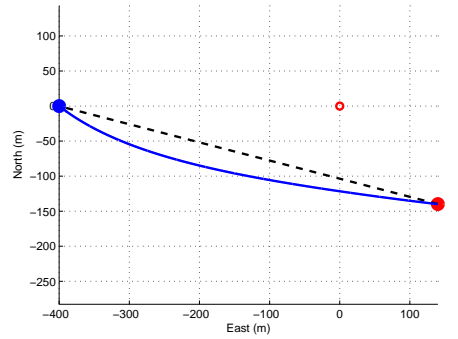

(h) Top View - No Bias Correction

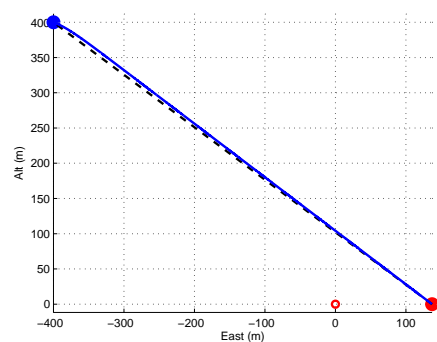

(c) Side View - High Gain Bias Correction

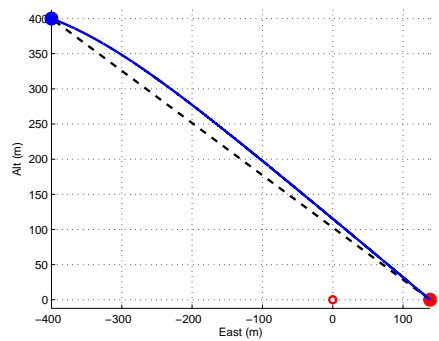

(f) Side View - Low Gain Bias Correction

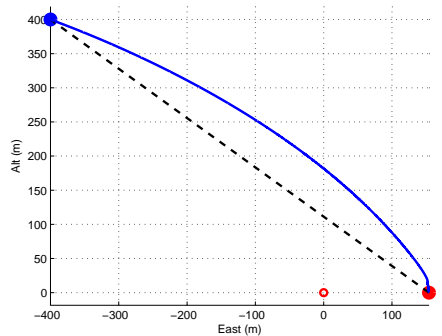

(i) Side View - No Bias Correction

Figure 10. Comparison of trajectories generated using feeback control with high gain bias correction (top row), the feeback control with low gain bias correction (middle row), and feeback control without bias correction (bottom row). 
BYU) which which is equipped with a Rabbit $340029 \mathrm{MHz}$ processor, rate gyros, accelerometers, absolute and differential pressure sensors. The autopilot measures $3.8 \times 5.1 \times 1.9 \mathrm{~cm}$ and weighs 17 grams.

The second frame in Figure 11 shows one of the airframes used for the flight tests reported in this thesis. The airframe is a flying wing with expanded payload bay and servo-driven elevons designed by the BYU Magicc Lab. It has a wingspan of $152 \mathrm{~cm}$, a length of $58 \mathrm{~cm}$, and a width of $12 \mathrm{~cm}$. It weighs $1.1 \mathrm{~kg}$ unloaded and $2.0 \mathrm{~kg}$ fully loaded. It is propelled by a brushless electric motor which uses an electronic speed control and is fueled by four multi-cell lithium polymer batteries. Typical speeds for the aircraft are between 15 and 20 meters per second (33 and 45 miles per hour). Maximum flight time for this aircraft is between 1 and 2 hours depending on external conditions and the mission it is required to fly.

The third frame in Figure 11 shows the ground station components. A laptop runs the Virtual Cockpit software that interfaces through a communication box to the MAV. An RC transmitter is used as a stand-by fail-safe mechanism to ensure safe operations.

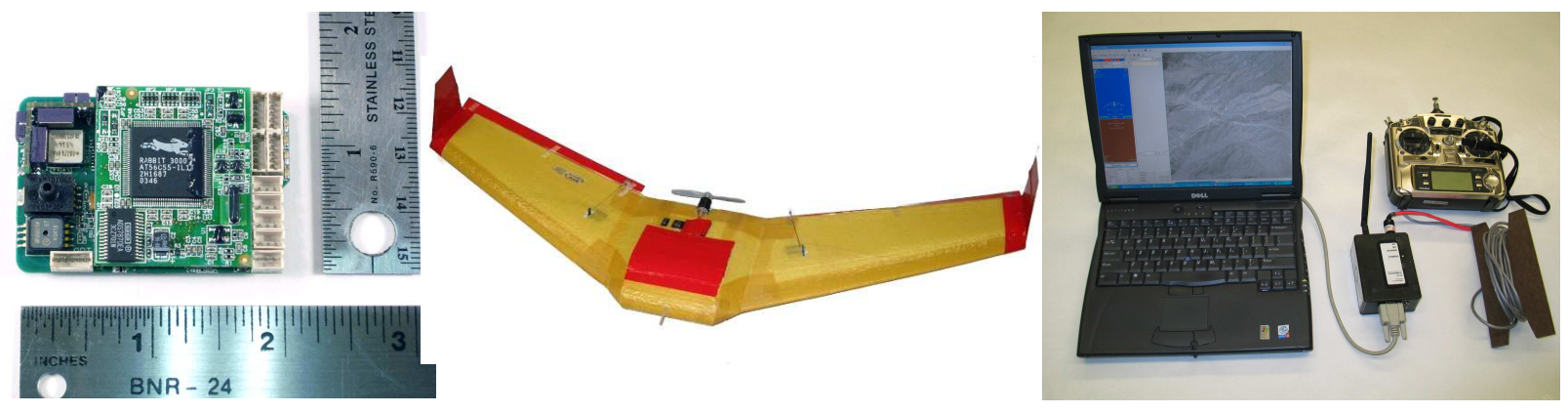

Figure 11. (a) Procerus' Kestrel autopilot. (b) MAV airframe. (c) Ground station components.

The vision processing platform is shown in Figure 12. The Helios platform is a small computing platform designed at BYU for real-time image processing in small autonomous vehicles. At the heart of the platform is a Xilinx Virtex-4 FX FPGA. The FPGA includes a PowerPC processor core as well as sophisticated reconfigurable logic. This platform combines the flexibility of a high-performance, embedded processor core with custom image processing hardware. This vision processing platform meets the size, weight, and power requirements for use in hand-launchable MAVs.

\section{B. Results}

The control law given by Equations (65) and (66) were tested using the hardware platfrom described in Section A. The vision processing was done at full frame rate $(30 \mathrm{~Hz})$, but the control was only updated at 5 $\mathrm{Hz}$. This update rate was chosen to demonstrate robustness to relatively slow update rates which would be required were the vision processing done offboard.

The control law was tested over a number of days with varying wind conditions. Although testing was not done using a moving target due to safety concerns, it is important to remember that the effects of wind and the effects of moving targets are essentially the same. Flight data from two flights in very different wind conditions are shown in Figures 13 and 14

Figure 13 shows flight data from a flight in which the initial MAV landing coordinate was $30 \mathrm{~m}$ east of the target, and there was a tailwind at $80 \%$ of the commanded airspeed. A strong tailwind represents one of the more challenging scenarios for the vision-based landing algorithm because of the corresponding increase in MAV groundspeed. The increased groundspeed significantly reduces the amount of time between initial target acquisition and impact. For the data set shown there were only 15 samples between intial acquisition and impact. This is compared to the 50 samples between intial acquisition and impact for the data set shown in Figure 14. The relatively short time between initial acquisition and impact makes it difficult to estimate the biases in the system. Despite this difficulty, the control algorithm was able to repeatedly guide the MAV to within 0 to 5 meters of the target.

Examination of Figure 13 shows the large corrections that were required to guide the MAV to the target site. This can be seen from the aggressive change in flight path beginning at the point where the target was intially acquired. The fact that the pitch commands shown in Figure 13(d) are continually decreasing indicates that the longitudinal misalignment correction should be negative. The total longitudinal 


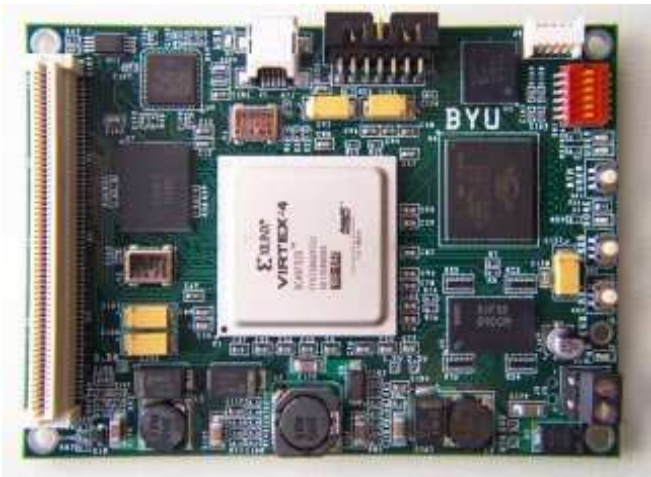

(a) Helios

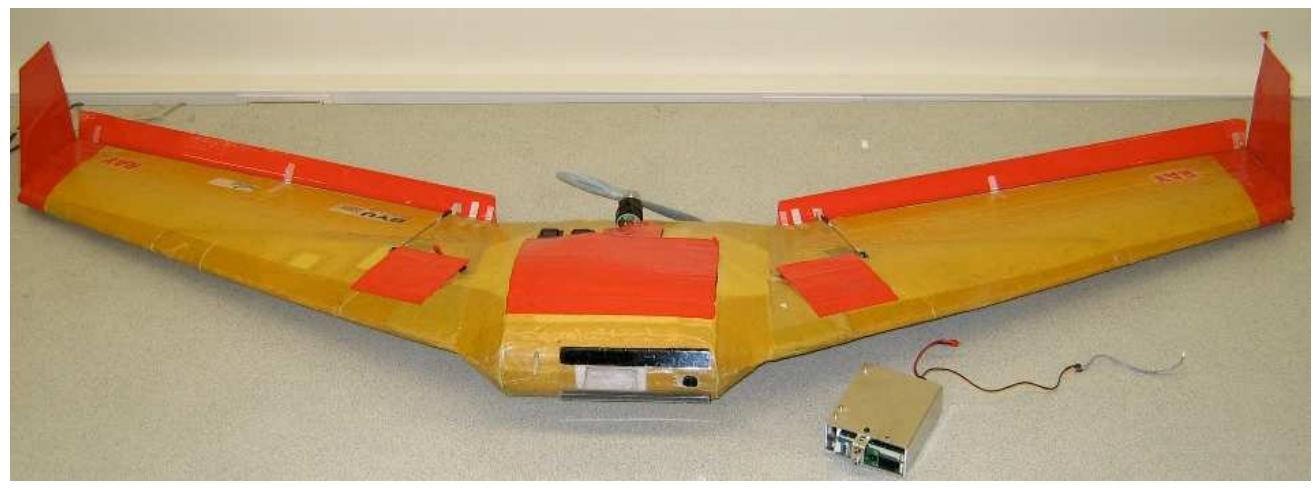

(b) Helios board shown next to the MAV used for flight testing

Figure 12. Flight test platform.

misalignment correction is given by the sum of $M$ and $N_{l o n}$ which indeed is negative, although the gains were not large enough to allow this term to make a meaningful difference. The continually increasing course commands shown in Figure 13(d) indicate the lateral misalignment correction should be positive. Once again the sign of the bias correction term is accurate, although the gains should have been higher to allow this term to have greater effect.

Based on the results shown in Figure 13, the bias estimation gains were increased by an order of magnitude, and more attempts at vision guided landing were attempted on a subsequent day. The wind conditions on this second day of testing resulted in a relatively mild headwind at approximately $15 \%$ of commanded airspeed. Results from one of the flight tests done on the second day of testing are shown in Figure 14. In this test the MAV was given an initial landing point $20 \mathrm{~m}$ north and $20 \mathrm{~m}$ east of the true target location. It can be seen from Figure 14 that immediately upon acquiring the target the MAV pulled up and to the south. This is the correct response since it was heading to a landing point that was short of the target and to the north.

Examining the MAV trajectory in the longitudinal plane (Figure 14(b)) shows that the glideslope after target acquisition and intial correction was fairly linear. This indicates that there was relatively little total longitudinal misalignment. This is borne out by the fact that the total longitudinal misalignment $\left(M+N_{\text {lon }}\right)$ hovers around zero. It is interesting to note that though there is significant parameter estimation error (given by $M$ ) and significant relative orientation estimation error (given by $N_{l o n}$ ) these errors essentially cancel each other even as they adapt.

In the lateral plane, the fact that $\chi^{c}$ is continually decreasing in Figure 14(d) indicates that the lateral bias correction term should be negative. This indeed is the case. Furthermore, because of the order of magnitude increase in bias estimation gains, this term was able to play a significant role in reducing deviation from the nominal linear trajectory. This in turn contributed to more accurate landing at the target site.

In total, 15 attempts at vision-guided landing were made on the second day of testing using the higher bias estimation gains. Of these attempts, twelve of the fifteen resulted in impact with the target. Of the three attempts that missed, each touched down within $5 \mathrm{~m}$ of the target. The algorithm was also used to 


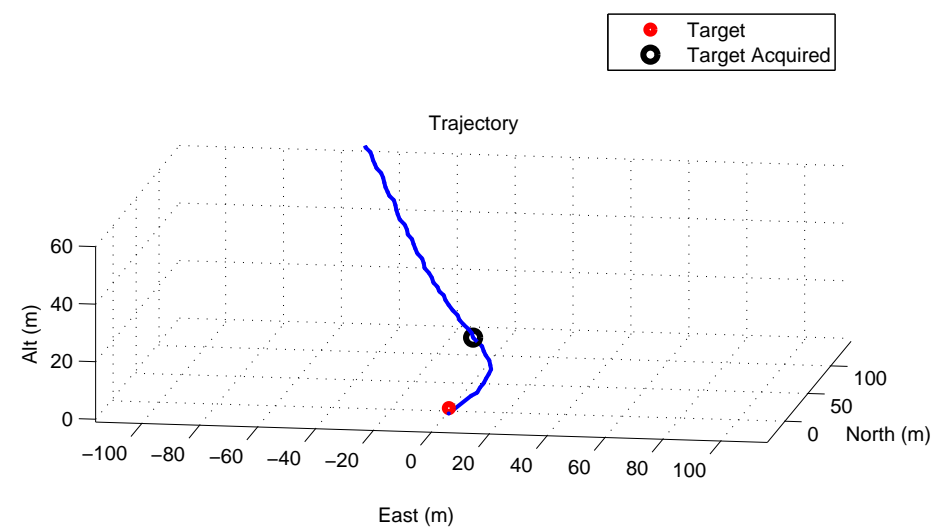

(a) 3D View

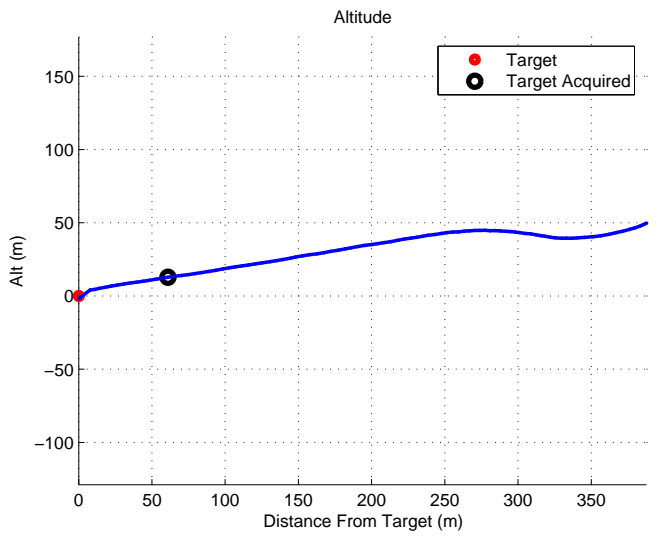

(b) Side View

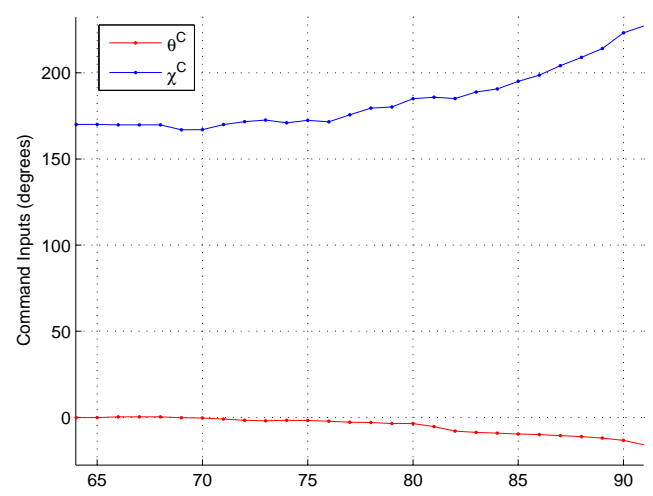

(d) Commands

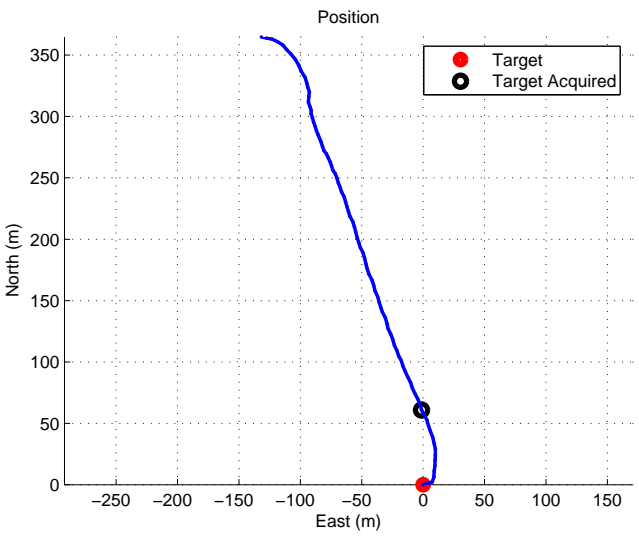

(c) Overhead View

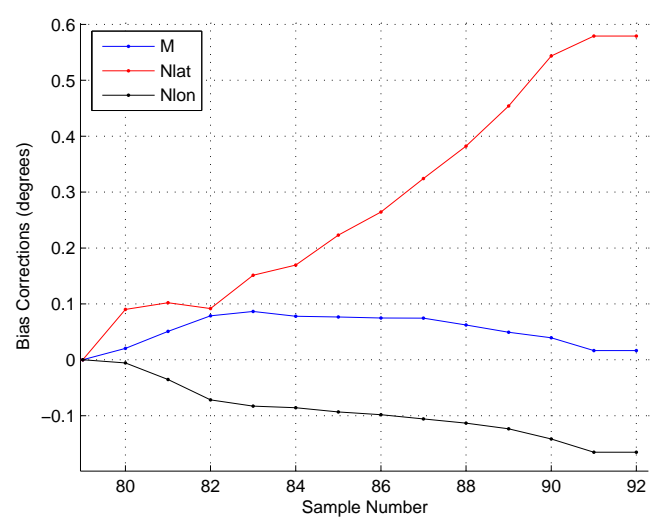

(e) Bias Correction Terms

Figure 13. Flight test results for landing on a stationary target in the presence of a tailwind at $80 \%$ of commanded airspeed. 


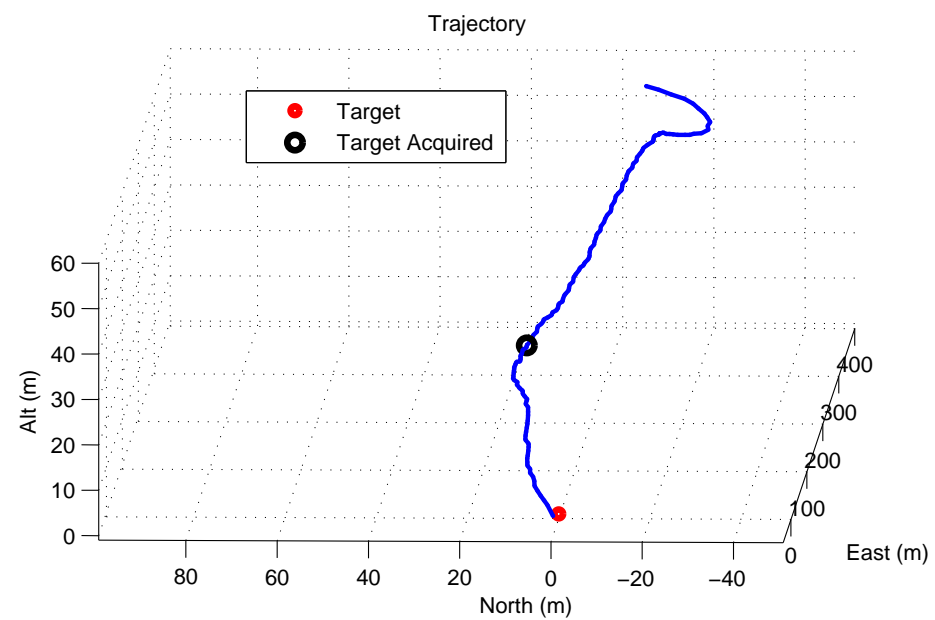

(a) 3D View

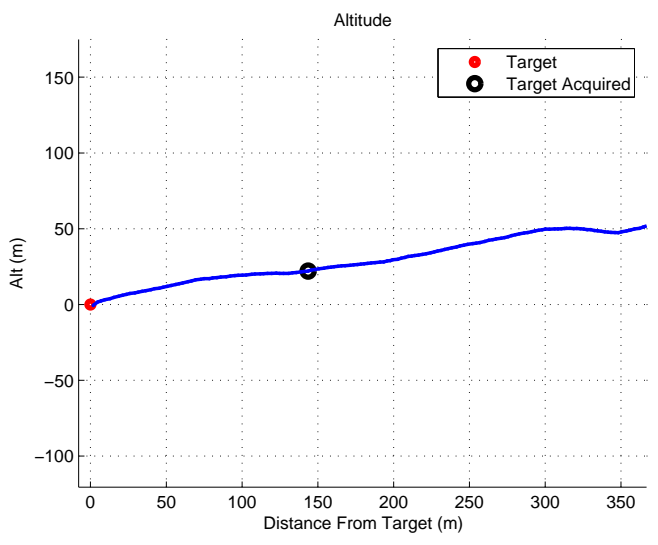

(b) Side View

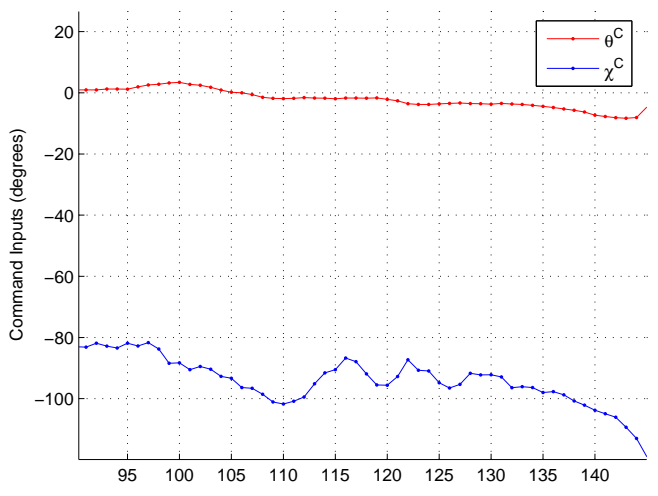

(d) Commands

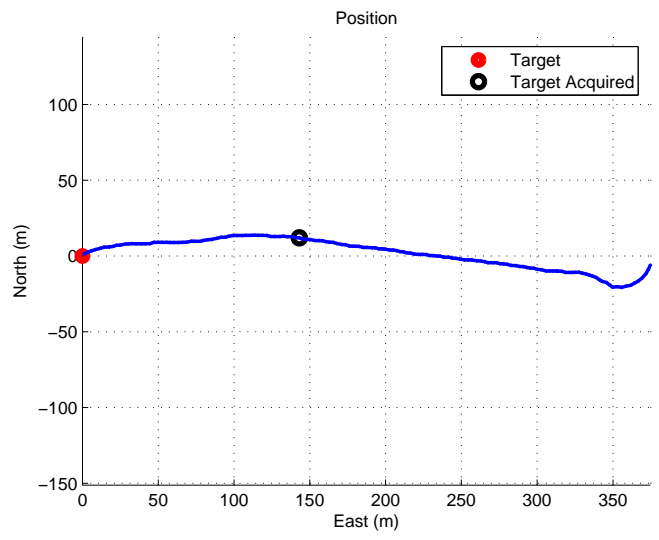

(c) Overhead View

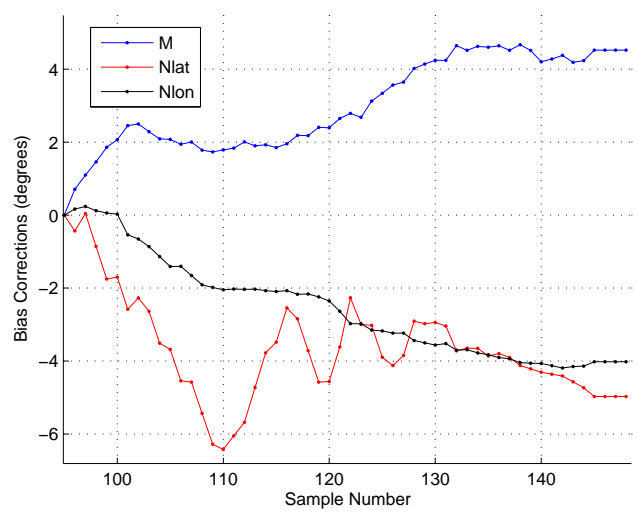

(e) Bias Correction Terms

Figure 14. Flight test results for landing on a stationary target in the presence of a headwind at $15 \%$ of commanded airspeed. 
guide the MAV to a successful landing in the back of a pickup truck moving at an approximately known velocity. Still images captured using the Helios platform during a landing attempt on the stationary target are shown in Figure 15. A sequence of images from the video footage of landing on a moving target are shown in Figure 16

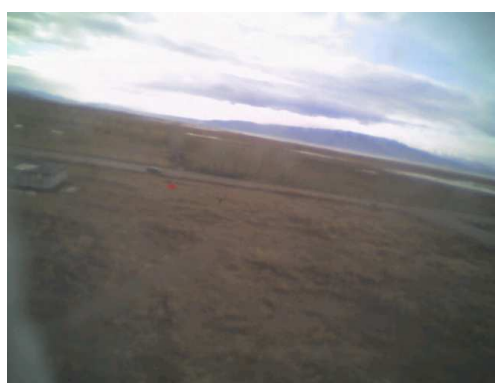

(a) $\approx 100 \mathrm{~m}$ from target

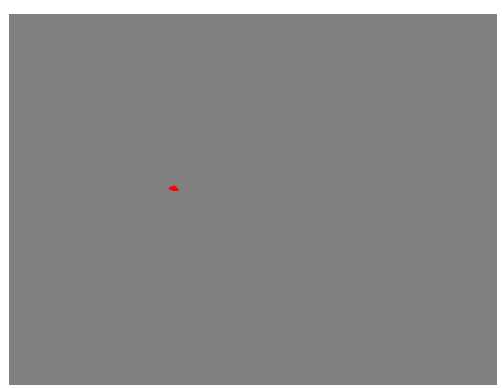

$(\mathrm{d}) \approx 100 \mathrm{~m}$ from target

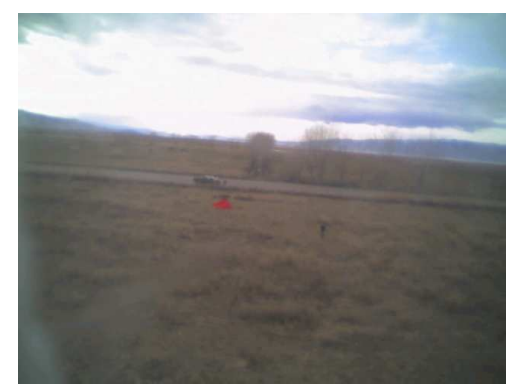

(b) $\approx 50 \mathrm{~m}$ from target

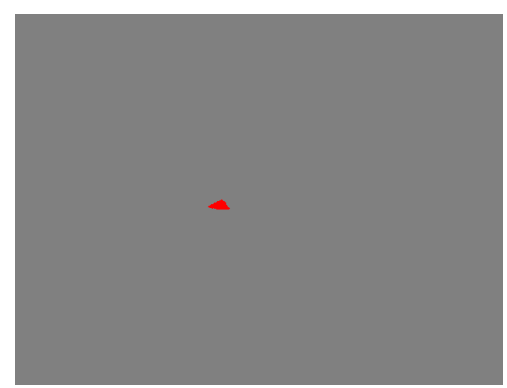

(e) $\approx 50 \mathrm{~m}$ from target

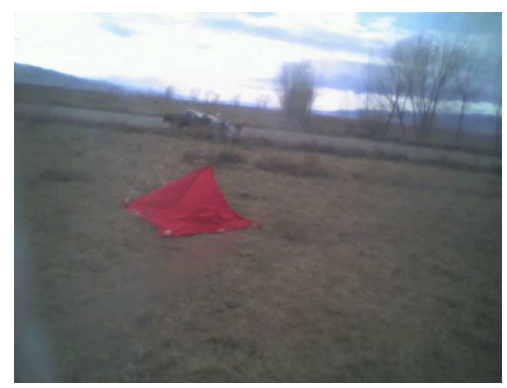

(c) $\approx 15 \mathrm{~m}$ from target

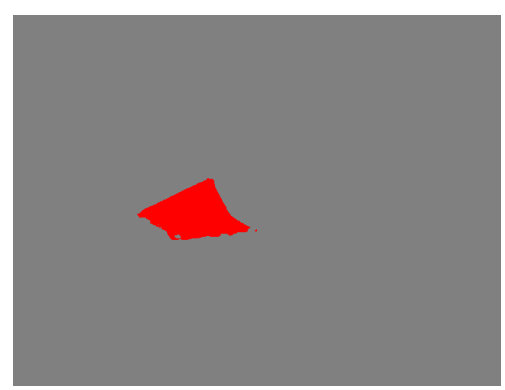

(f) $\approx 15 \mathrm{~m}$ from target

Figure 15. Digital images of the target as captured by the Helios. The top row shows the raw images. The bottom row shows the processed images.

\section{Conclusion}

A control law was developed for using vision-based feedback to accurately land on moving targets in the presence of wind and bias errors. It was shown that for sufficiently small bias errors vision-based feedback without bias estimation would result in accurate landing on a moving target. However, because bias errors can be introduced from a number of sources including relative orientation estimation error, parameter estimation error, wind estimation error, and target velocity estimation error, a scheme for estimating and correcting for bias errors was introduced. This bias correction scheme was shown to significantly reduce the deviations from the nominal linear glideslope caused by bias errors. The control scheme was tested in hardware flight tests using onboard vision processing and a stationary target. The control was deliberately run at only $5 \mathrm{~Hz}$ to demonstrate robustness to relatively slow update rates. The control scheme was able to guide the MAV to impact with a $2 \mathrm{~m}$ square target on 12 of 15 attempts. On all attempts the MAV touched down within $5 \mathrm{~m}$ of the target center. The method was also used to successfully land a MAV in the back of a pickup truck moving at an approximately known velocity. The algorithm demonstrated similar performance under a variety of wind conditions including significant tailwinds, crosswinds, and headwinds.

The proposed method is valid for both fixed and gimbaled cameras. However, a fixed camera was used for all reported flight tests. The use of a fixed camera requires a wide angle lens to keep the object of interest in the field of view, especially in high wind conditions where the MAV may have a significant crab angle. Performance could be improved by using a gimbaled camera with a narrow angle lens. This would allow the target to be acquired at a greater range, and ensure that the target is able to be maintained in the sensor field of view. 


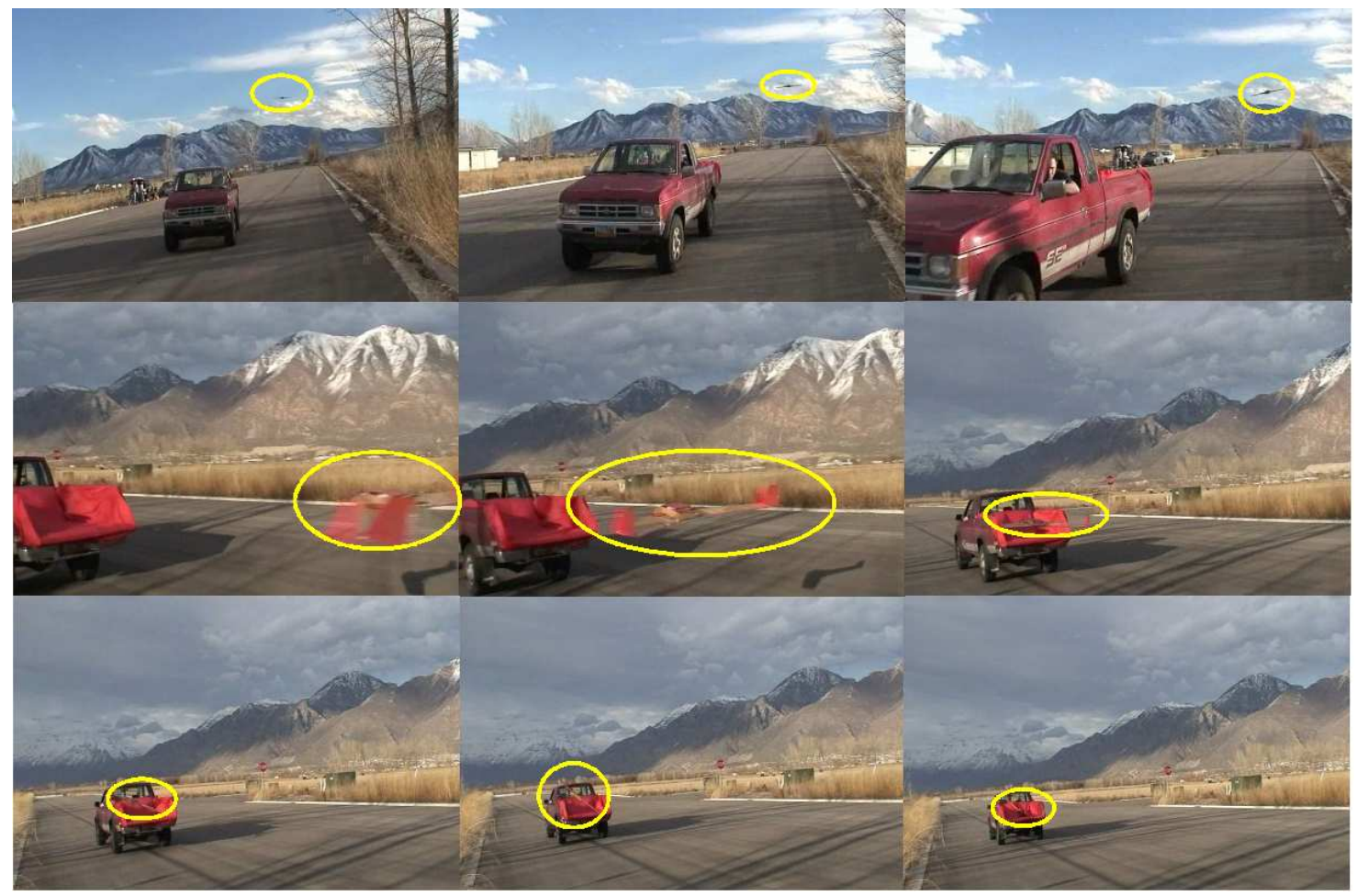

Figure 16. Sequence of images showing successful landing on a moving platform.

\section{References}

${ }^{1}$ Barber, D. B., Redding, J. D., McLain, T. W., Beard, R. W., and Taylor, C. N., "Vision-based Target Geo-location using a Fixed-wing Miniature Air Vehicle," Journal of Intelligent and Robotic Systems, Vol. 47, No. 4, December 2006 , pp. 361-382.

${ }^{2}$ Saripalli, S., Montgomery, J., and Sukhatme, G., "Visually guided landing of an unmanned aerial vehicle," Robotics and Automation, IEEE Transactions on, Vol. 19, No. 3, 2003, pp. 371-380.

${ }^{3}$ Shakernia, O., Vidal, R., Sharp, C. S., Ma, Y., and Sastry, S., "Multiple View Motion Estimation and Control for Landing an Unmanned Aerial Vehicle," Proceedings of the 2002 IEEE International Conference on Robotics 85 Automation, Washington DC, May 2002, pp. 2793-2798.

${ }^{4}$ Saripalli, S., Montgomery, J., and Sukhatme, G., "Vision-based autonomous landing of an unmanned aerial vehicle," Robotics and Automation, 2002. Proceedings. ICRA'02. IEEE International Conference on, Vol. 3, 2002.

${ }^{5}$ Saripalli, S. and Sukhatme, G., "Landing on a Moving Target using an Autonomous Helicopter," Proceedings of the International Conference on Field and Service Robotics, July, 2003.

${ }^{6}$ Hintze, J., Christian, D., Theodore, C., Tischler, M., and McLain, T., "Automated Landing of a Rotorcraft UAV in a Noncooperative Environment," Proceedings of the 60th Annual Forum of the American Helicopter Society, Baltimore, Maryland, June 2004

${ }^{7}$ Hintze, J., Autonomous Landing of a Rotary Unmanned Aerial Vehicle in a Non-Cooperative Environment Using Machine Vision, Master's thesis, Brigham Young University, April 2004.

${ }^{8}$ Yakimenko, O., Kaminer, I., Lentz, W., and Ghyzel, P., "Unmanned aircraft navigation for shipboard landing using infrared vision," Aerospace and Electronic Systems, IEEE Transactions on, Vol. 38, No. 4, 2002, pp. 1181-1200.

${ }^{9}$ Frezza, R. and Altafini, C., "Autonomous landing by computer vision: an application of pathfollowing in SE (3)," Decision and Control, 2000. Proceedings of the 39th IEEE Conference on, Vol. 3, 2000.

${ }^{10}$ Chahl, J., Srinivasan, M., and Zhang, S., "Landing Strategies in Honeybees and Applications to Uninhabited Airborne Vehicles," The International Journal of Robotics Research, Vol. 23, No. 2, 2004, pp. 101-110.

${ }^{11}$ Barrows, G. and Neely, C., "Mixed-mode VLSI optic flow sensors for in-flight control of a micro air vehicle," Proceedings SPIE, San Diego, August 2000, pp. 52-63.

${ }^{12}$ Zufferey, J.-C. and Floreano, D., "Toward 30-gram autonomous indoor aircraft: Vision-based obstacle avoidance and altitude control," Proceedings of the 2005 IEEE International Conference on Robotics 85 Automation, Barcelona, April 2005, pp. 2594-2599.

${ }^{13}$ Ruffier, F. and Franceschini, N., "Visually Guided Micro-Aerial Vehicle: automatic take off, terrain following, landing and wind reaction," Proceedings of the 2004 IEEE International Conference on Robotics 85 Automation, New Orleans, 2004, pp. 2339-2346.

${ }^{14}$ Barrows, G. L., Chahl, J. S., and Srinivasan, M. V., "Biomimetic Visual Sensing and Flight Control," The Aeronautical Journal, London: The Royal Aeronautical Society, Vol. 107, No. 1069, 2003, pp. 159-168. 
${ }^{15}$ Barber, D., Griffiths, S., McLain, T., and Beard, R., "Autonomous Landing of Miniature Aerial Vehicles," Infotech@ Aerospoace, 2005, pp. 1-14.

${ }^{16}$ Beard, R., Kingston, D., Quigley, M., Snyder, D., Christiansen, R., Johnson, W., McLain, T., and Goodrich, M., "Autonomous Vehicle Technologies for Small Fixed Wing UAVs," AIAA Journal of Aerospace Computing, Information, and Communication, Vol. 2, No. 1, January 2005, pp. 92-108. 